\section{Personality traits and gender: effects on self-efficacy at work}

\section{ÜẨE CONICET}

Paulina Robalino, Lic. UADE - CONICET

probalino@uade.edu.ar
Mariel Musso, PhD.

National Research Council-CIIPME CONICET mariel.musso@hotmail.com
XVI European Congress of Psychology JULY 2-5 2019 MOSCOW, RUSSIA

\section{BACKGROUND}

- Personality traits represent stable individua characteristics that mostly derive from individual genetic endowment.

- Self-efficacy can enhance learning and performance (Caprara et al., 2010).

Self-efficacy is a crucial cognitive and motivational belief impacting on several aspects of behavior at workplace.

- Self-efficacy has been related to the effort and perseverance, coping strategies to deal with labor demands, work engagement, and job performance.

The aim was to analyze the effects and

(2) interaction effects of personality trails and gender on self-efficacy at work.

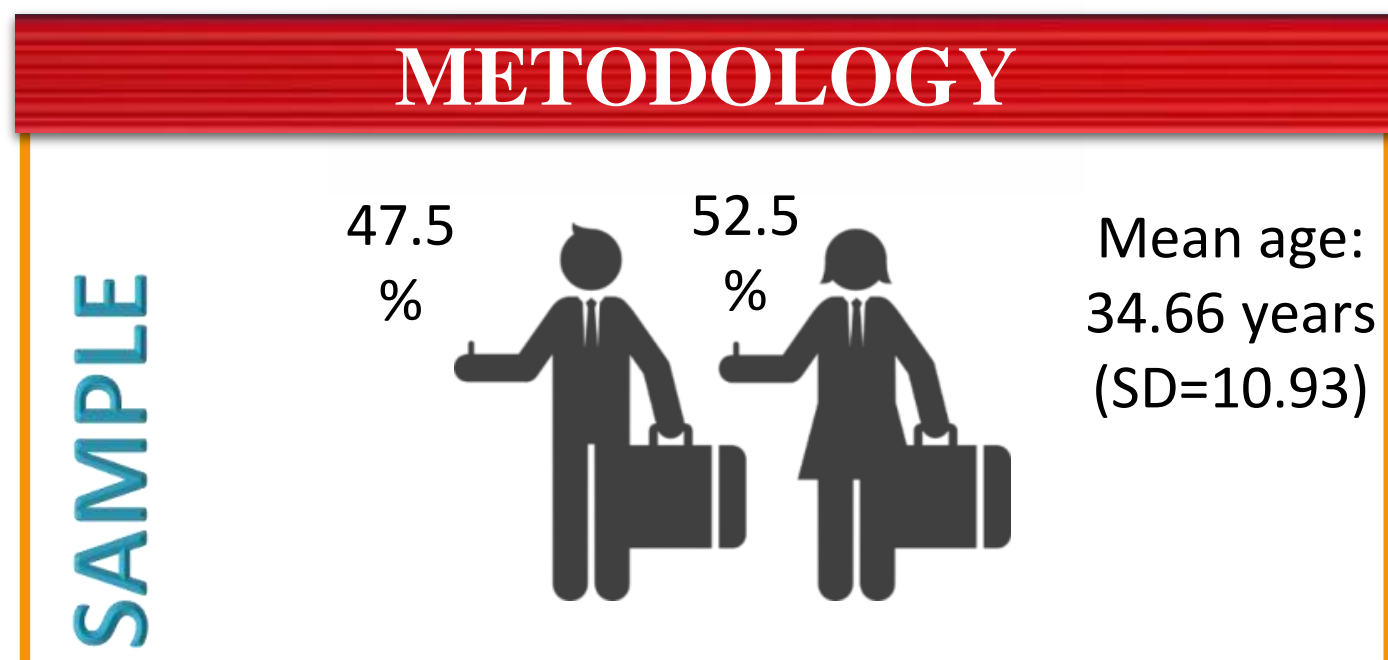

314 employees from three different sectors (blue, white and pink collar)

\section{INSTRUMENTS}

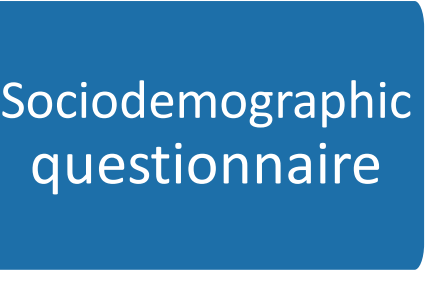

Spanish
version of Self-
efficacy at
work scale

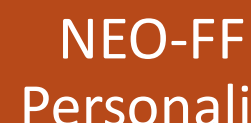

Personality

Inventory

Factorial design

DESING trail) $\times 2$ (gender) was applied.

\title{
RESULTS
}

Neuroticism, Conscientiousness, Extraversion, Agreeableness on task, Social, Emotional Self-Oriented, Emotional Other-oriented to help self-efficacy.

Table 1. Effect of Personality on Task Self-efficacy $M(S D) \quad F(312)$

Openness to Low $24.72(3.80) \quad 2.594 \quad 0.008$

Neuroticism

$\begin{array}{ll}\text { Low } & 24.72(3.80) \\ \text { Higth } & 25.39(3.37)\end{array}$

Low $26.65(2.99)$

Higth $23.89(3.63)$

$\begin{array}{llll}\text { Conscientiousness } & \text { Low } & 23.71(3.38) \\ \text { Higth } & 26.37(3.40) & 47.856 * * * & 0.133\end{array}$

ow $23.50(3.74)$

Extraversion

Higth $25.54(3.45)$ 20.348*** 0.061

Low $24.17(3.71)$

\begin{tabular}{llll} 
Agreeableness & Higth $25.58(3.48)$ & $11.745 * *$ & 0.036 \\
\hline
\end{tabular} able 4. Effect of

$\begin{array}{lccc} & \mathrm{M}(\mathrm{SD}) & \mathrm{F}(312) & \eta^{2}\end{array}$

$\begin{array}{lllll} & \text { Low } & 7.93(1.68) & 2.381 & 0.008\end{array}$

$\begin{array}{lllll}\text { Neuroticism Low } 8.31(1.60) & \text { 5.638* } & 0.018\end{array}$

Higth $7.87(1.62)$

$\begin{array}{lcccc}\text { Conscientiousness } & \text { Low } & 7.65(1.68) & \text { 21.669*** } & 0.065 \\ & \text { Higth } & 8.47(1.43) & & \end{array}$

Low $7.55(1.77) \quad 11.082 * * \quad 0.034$

Extraversion $\quad$ Higth $8.23(1.53)$ 11.082** 0.034

Low $7.75(1.64)$

Higth $8.26(1.57) \quad 7.371^{* *} \quad 0.023$

Table 5. Effect of Personality on Self-efficacy in recognizing the emotions of others

\begin{tabular}{lcccc}
\hline & & $\mathrm{M}(\mathrm{SD})$ & $\mathrm{F}(312)$ & $\eta^{2}$ \\
\hline Openness to & Low & $7.81(1.44)$ & & $\mathbf{6}$ \\
Experience & Higth & $8.25(1.47)$ & $\mathbf{6 4 3} *$ & 0.022 \\
Neuroticism & Low & $8.17(1.53)$ & & \\
& Higth & $7.88(1.41)$ & 2.876 & 0.009 \\
Conscientiousness & Low & $7.68(1.45)$ & $15.46^{* * *}$ & 0.048 \\
& Higth & $8.33(1.42)$ & & \\
Extraversion & Low & $7.86(1.41)$ & 0.961 & 0.061 \\
& Higth & $8.05(1.49)$ & & \\
Agreeableness & Low & $7.84(1.50)$ & 2.552 & 0.008 \\
& Higth & $8.11(1.43)$ & & \\
\hline
\end{tabular}

\begin{tabular}{|c|c|c|c|c|}
\hline & & $M(S D)$ & $F(312)$ & $\eta^{2}$ \\
\hline $\begin{array}{l}\text { Openness to } \\
\text { Experience }\end{array}$ & $\begin{array}{l}\text { Low } \\
\text { Higth }\end{array}$ & $\begin{array}{l}20.29(3.40) \\
20.78(2.96)\end{array}$ & 1.758 & 0.006 \\
\hline Neuroticism & $\begin{array}{l}\text { Low } \\
\text { Higth }\end{array}$ & $\begin{array}{l}21.65(2.63) \\
19.72(3.36)\end{array}$ & $29.28^{* * *}$ & 0.086 \\
\hline Conscientiousness & $\begin{array}{l}\text { Low } \\
\text { Higth }\end{array}$ & $\begin{array}{l}19.59(3.34) \\
21.45(2.80)\end{array}$ & $28.302^{* * *}$ & 0.030 \\
\hline Extraversion & $\begin{array}{l}\text { Low } \\
\text { Higth }\end{array}$ & $\begin{array}{l}18.57(3.84) \\
21.18(2.66)\end{array}$ & $45.44^{* * *}$ & 0.127 \\
\hline Agreeableness & $\begin{array}{l}\text { Low } \\
\text { Higth }\end{array}$ & $\begin{array}{l}19.28(3.52) \\
21.32(2.72)\end{array}$ & $33.321^{* * *}$ & 0.096 \\
\hline
\end{tabular}

Table 3. Effect of Personality on Emotional Self-oriented Selfefficacy

\begin{tabular}{lcccc} 
& & $M(S D)$ & $F(312)$ & $\eta^{2}$ \\
\hline Openness to & Low & $11.51(2.44)$ & 3.610 & 0.011 \\
Experience & Higth & $12.03(2.30)$ & & \\
Neuroticism & Low & $12.43(2.14)$ & $19.332^{* * *}$ & 0.058 \\
& Higth & $11.25(2.45)$ & & \\
Conscientiousness & Low & $11.13(2.29)$ & $\mathbf{2 1 . 9 3 * * *}$ & 0.066 \\
& Higth & $12.35(2.35)$ & & \\
Extraversion & Low & $11.03(2.78)$ & $\mathbf{9 . 6 6 * *}$ & 0.030 \\
& Higth & $11.97(2.20)$ & & \\
Agreeableness & Low & $11.19(2.56)$ & $\mathbf{1 1 . 0 3 3 * *}$ & 0.034 \\
& Higth & $12.09(2.21)$ & & \\
\hline & & & &
\end{tabular}

Men have greater task selfefficacy $(\mathrm{t}(312)=-2.730 ; \mathrm{p}<.01)$ and social self-efficacy $(t(312)=-2.793$; $\mathrm{p}<.01)$.

There are no gender differences in self-oriented emotional selfefficacy, emotional self-efficacy to understand-other, emotional selfefficacy to help others

Openness to Experience and

Conscientiousness have a significant effect on "self-efficacy in recognizing the emotions of others"

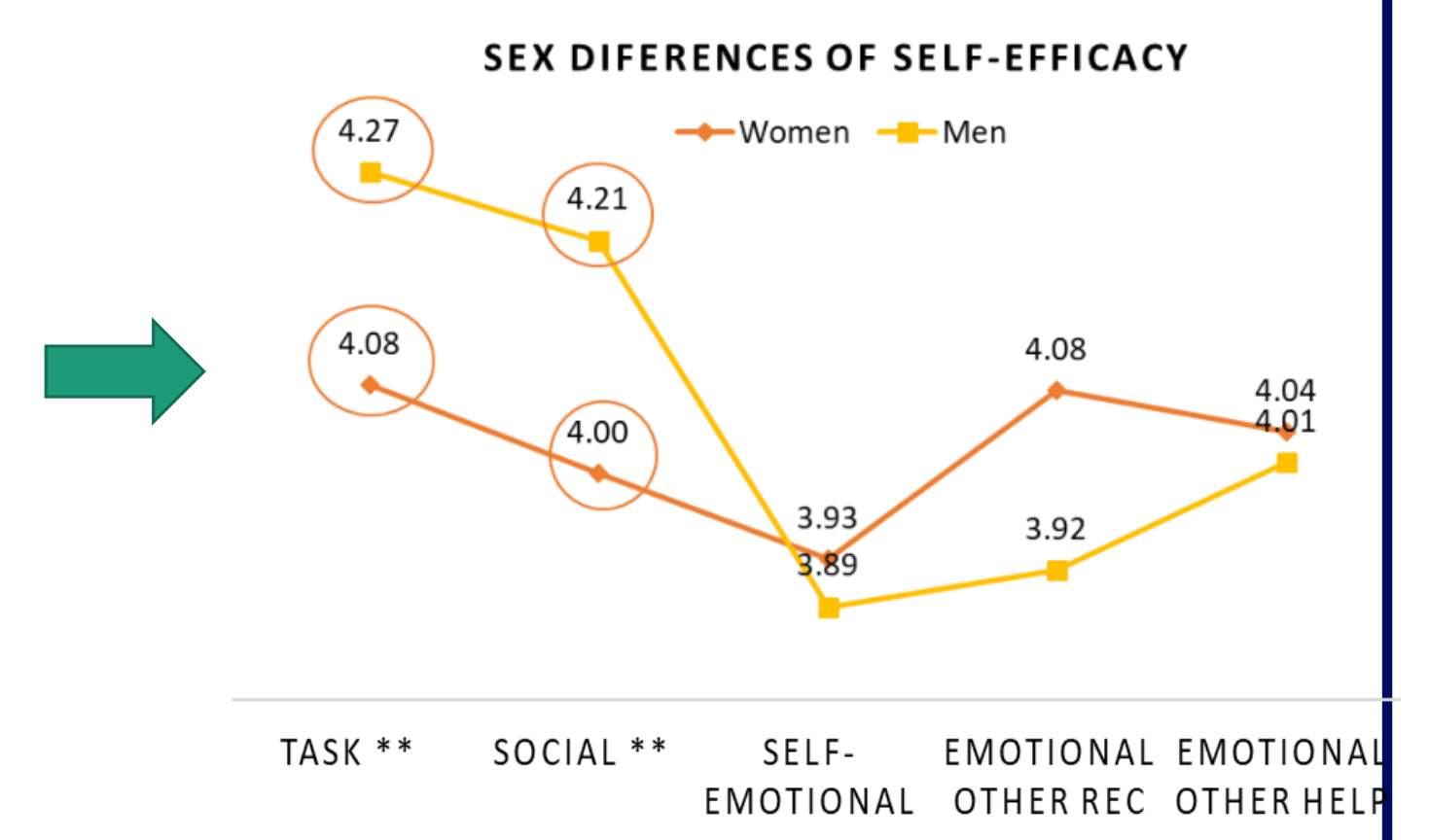

MANOVA's results suggest that there are no interactions effects between gender and personality traits on self-efficacy at work.

\section{DISCUSSION} Our results are consistent with previous studies (De Miguel et al. 2005 ).
Personality traits have a significant effect on Self-efficacy. Even we do not found interaction, there are differences in Self-efficacy by gender.

Future research should include a performance measure.
*. Significative at level $<.05$ $* *$. Significative at level $<.01$ $* * *$. Significative at level $<.001$ 